\title{
$-1$

\section{ANÁLISIS DIDÁCTICO DE LA EDUCACIÓN PATRIMONIAL EN ALUMNADO DE CIENCIAS DE LA EDUCACIÓN}

\author{
Antonio Nadal Masegosa \\ ORCID: https://orcid.org/0000-0002-2788-0058 \\ Departamento de Teoría e Historia de la Educación y Métodos de Investigación y Diagnóstico en Educación. \\ Facultad de Ciencias de la Educación. \\ Universidad de Málaga. \\ antonionm@uma.es \\ Lucía Rodríguez Olay \\ ORCID: https://orcid.org/0000-0002-3704-9962 \\ Departamento de Ciencias de la Educación, área de Didáctica de la Lengua y la Literatura. \\ Facultad de Formación del Profesorado y Educación. \\ Universidad de Oviedo \\ rodriguezolucia@uniovi.es
}

Para citar este artículo puede utilizar el siguiente formato:

Antonio Nadal Masegosa y Lucía Rodríguez Olay (2021): "Análisis didáctico de la educación patrimonial en alumnado de ciencias de la educación", Revista DELOS, Vol 14 № 38 (junio 2021). En línea:

https://www.eumed.net/es/revistas/delos/vol-14-no-38-junio-2021/educacion-patrimonial

\section{RESUMEN}

La formación en educación patrimonial es básica para la conservación, conocimiento y aprecio de lo que nos pertenece. El hecho de que ella no existiera en la educación primaria, secundaria y universitaria supondría una carencia de consecuencias considerables, por lo cual, en el marco del Proyecto de Innovación Educativa (PIE) 19-036, Implementación de mejoras en el proceso de enseñanza-aprendizaje de la Educación Patrimonial en Humanidades, Ciencias Sociales y Educación, aprobado por la Universidad de Málaga para el bienio 2019/2021, a través de dos cursos académicos, se ha trasladado a la totalidad del alumnado del Doble Grado en Educación Primaria y Estudios Ingleses, y al de dos cursos del Grado en Educación Social, el cuestionario elaborado por el citado PIE, para conocer el nivel que poseen en la materia, lo cual debe ir acompañado por el conocimiento de la relevancia que la legislación actual otorga a la materia de estudio, pues sin una base fundamental establecida desde la infancia, sería difícil construir un conocimiento tan siquiera mínimo sobre qué es y qué implica la educación patrimonial. El análisis didáctico que se desarrolla se complementa, para efectuar la pertinente triangulación, con las opiniones del alumnado en torno a su baja formación reconocida, complementándose con la demostración de la práctica absoluta ausencia de materias de educación patrimonial en los grados de la Facultad de Ciencias de la Educación de la 
Universidad de Málaga, hecho contrastable gracias a sus memorias de verificación de los títulos correspondientes.

Palabras clave: cultura científica, enseñanza, formación, patrimonio, universidad

\section{DIDACTIC ANALYSIS OF HERITAGE EDUCATION IN EDUCATIONAL SCIENCE STUDENTS}

\section{ABSTRACT}

Training in heritage education is basic for the conservation, knowledge and appreciation of what belongs to us. The fact that it could not exist in primary, secondary and university education would imply a lack of considerable consequences, for which, within the framework of the Educational Innovation Project (PIE) 19-036, Implementation of improvements in the teaching-learning process of Heritage Education in Humanities, Social Sciences and Education, approved by the University of Malaga for the 2019/2021 biennium, through two academic years, has been transferred to all students of the Double Degree in Primary Education and English Studies, and to two courses of the Degree in Social Education, the questionnaire prepared by the aforementioned PIE, to know the level they have in the matter, which must be accompanied by knowledge of the relevance that current legislation gives to the subject of study, because without a fundamental base established from childhood, it would be difficult to build even a minimal knowledge about what heritage education is and what it implies. The didactic analysis that is developed is complemented, in order to carry out the relevant triangulation, with the opinions of the students regarding their low recognized training, complemented with the demonstration of the almost absolute absence of heritage education subjects in the degrees of the Faculty of Sciences of Education of the University of Malaga, verifiable fact thanks to the verification reports of the corresponding degrees.

Keywords: scientific culture, teaching, training, heritage, university.

\section{INTRODUCCIÓN}

En la última década, el interés por la educación patrimonial está en auge. Desde ponencias en congresos internacionales (Arjones, 2017), hasta la celebración, durante cuatro años consecutivos, de congresos internacionales sobre patrimonio, en la Universidad de Málaga, o de las Jornadas Europeas de Patrimonio del año 2020 en Vizcaya -evento cultural de difusión del patrimonio con mayor alcance y participación en Europa (De la Hoz, 2021)-, pasando por la publicación de artículos relacionados en revistas de reconocido prestigio (Clarembeaux, 2010; Fontal, García, Arias y Arias, 2019; Palomero-llardia, 2020), por citar algunos ejemplos, hacen ver que debiera considerarse esta disciplina como emergente y de atención creciente.

La investigación científica en ciencias sociales debe tener su fundamento en las fuentes primarias de conocimiento, y los resultados obtenidos no tienen por qué coincidir ni con las presunciones ni con los juicios de quienes investigan, puesto que, como podemos comprobar, distintos ámbitos académicos universitarios, en ocasiones, no mantienen una correlación directa con distintos hechos, tales como los contenidos en la enseñanza tanto obligatoria como post-obligatoria, o la formación del profesorado. Hemos de conocer, atendiendo a la información real, los hechos 
verídicos, alejándonos del mero discurso (Foucault, 2019), para que la ciencia no se constituya en algo de lo que defendernos (Feyerabend, 2001), sino en lo que apoyarnos, de forma crítica.

Los referentes disciplinarios y didácticos de la educación patrimonial son enormemente diversos: los paisajes naturales, culturales, rurales, urbanos; el patrimonio arqueológico; el patrimonio musical; la memoria histórica; el patrimonio inmaterial; el género y un enorme etcétera (Cambil y Tudela, 2017). La educación patrimonial no versa únicamente sobre el pasado, sino sobre la historia viva, pues nuestro día a día, como sabemos, está basado en el ayer, al igual que es la base del mañana.

La evolución y el estado actual de la investigación en educación patrimonial a través del análisis de indicadores de alto impacto, igualmente, ha sido considerado un tema de publicación en revistas de reconocido prestigio: nos encontraríamos ante un tránsito de área de conocimiento a disciplina científica (Fontal e Ibañez-Etxeberria, 2017). Contamos con evaluaciones de la cultura material de la escuela y la educación patrimonial (Escolano, 2010), y de la actualidad y tendencias en educación patrimonial (Ibañez, Fontal y Cuenca, 2015).

En el Estado español, existe la Ley 16/1985, de 25 de junio, del Patrimonio Histórico Español, en la cual se expresa que se impulsa una política adecuada para gestionar con eficacia el Patrimonio Histórico Español, que incluya el estímulo educativo. Con base en esta legislación, encontramos que se prevé que el Ministerio de Educación, Cultura y Deporte, a través del Instituto del Patrimonio Cultural de España, y las Comunidades Autónomas, entes locales, fundaciones, asociaciones u otras instituciones, contribuirán a la realización de diversas acciones en el ámbito de la educación patrimonial, que puedan ser incluidas en el Plan Nacional de Educación y Patrimonio (Carrión, 2015), un recurso que incluye unidades didácticas de patrimonio cultural inmaterial, recursos virtuales y tecnológicos -como juegos de ordenador-, proyectos educativos y didácticos... (Ministerio de Cultura y Deporte, 2021).

Diversos entes autonómicos, igualmente, desarrollan actuaciones en torno al tema que nos ocupa, como por ejemplo el Plan de Educación Patrimonial de la Comunidad de Madrid (2021), o el primer Massive Online Course Online (curso abierto y en línea) que desarrollaría el Instituto Andaluz del Patrimonio Histórico (2021), sobre Patrimonio Cultural de Andalucía, en colaboración con el Instituto Andaluz de Administración Pública, de abril a junio de 2021.

Pese al nivel alcanzado por la educación patrimonial, hemos de bajar al terreno, por decirlo de algún modo, trascender de lo general a lo particular, puesto que en el proceso histórico de esta sociedad, suceden hechos nuevos que provocan intentos de volver sobre sí misma (Freire, 2011), y es absolutamente necesaria la conjunción, por una parte, de los hechos particulares, como fundamento imprescindible, y por otra, de la dinámica global, como marco de funcionamiento en el que insertarlos (Lacomba, 2011). En definitiva, se pretende aproximar las experiencias formativas en educación patrimonial del alumnado de la Facultad de Ciencias de la Educación de la Universidad de Málaga con las relacionadas con su formación previa, estableciendo comparaciones, y añadiendo valiosa información cualitativa.

\section{MÉTODO}


La metodología empleada parte de la elaboración de temáticas concretas, y cuestionario, en el marco del Proyecto de Innovación Educativa PIE 19-036, Implementación de mejoras en el proceso de enseñanza-aprendizaje de la Educación Patrimonial en Humanidades, Ciencias Sociales y Educación, aprobado por la Universidad de Málaga para el bienio 2019/2021, cuya coordinadora es la Profesora Titular de Historia Contemporánea Mercedes Fernández Paradas. El diseño parte del objetivo de conocer, y mostrar, un estudio detallado, es decir, un análisis, pero que vaya más allá y, por tanto, no sea una mera descripción de resultados, sino que se constituya en propio, y adecuado, para enseñar a cualquier lector o lectora, es decir, que sea didáctico.

A través de un diseño multimétodo, no solo obtenemos los datos numéricos que logramos a través de los cuestionarios realizados, sino que procedemos a un análisis cualitativo a través de la información que nos proporciona la etnografía virtual (Hine, 2004), dentro de un paradigma que va más allá de la mera obtención de datos (Taylor y Bogdan, 2002). El objetivo principal de la investigación no es simplemente el análisis de resultados, sino que se amplía en la comparación de los mismos en dos cursos diferentes, así como en la descripción general del asunto en la normativa vigente y en las guías docentes de la Facultad de Ciencias de la Educación de la Universidad de Málaga. El análisis es aún más rico gracias al testimonio directo de decenas de estudiantes, a través de la herramienta del foro, del campus virtual interno de la Facultad de Ciencias de la Educación, una vez finalizados los cuestionarios, y dados los resultados de los cursos estudiados, estrategia realizada en el curso 2020/2021.

La población objeto de estudio es la totalidad del alumnado existente del Doble Grado en Educación Primaria y Estudios Ingleses de la Universidad de Málaga, de implantación en el curso 2019/2020, de dicho curso y del curso 2020/2021, ampliándose el tamaño de muestra con el alumnado de tercer curso de la asignatura obligatoria Política y Legislación Socioeducativa, del Grado en Educación Social, del curso 2020/2021, y con el alumnado de la asignatura optativa de cuarto curso de dicho grado llamada Inmigración, Minorías Étnicas, y Educación Intercultural, durante el curso 2019/2020. De 190 cuestionarios entregados, se obtendrían 141 realizados completamente.

Los cuestionarios realizados por parte del PIE 19-036, a través de Google Forms, constan de 49 ítems, de los cuales seleccionamos los ítems 7 a 29, obviando los referidos a características personales y a otras cuestiones. Se analizan los resultados de forma comparada, acompañando a dicho análisis el estudio, a través de la etnografía virtual, de la referencia a la educación patrimonial en la normativa legislativa vigente, y en las memorias de verificación de la totalidad de los grados de la facultad. El procedimiento empleado, como vamos a leer a continuación, parte de una descripción particular de resultados, para a continuación describir de forma general algunas de las causas de dichos resultados, finalizando con el comentario interpretativo pertinente, para ayudar a quien lea a establecer las conexiones apropiadas entre los detalles específicos y los argumentos más abstractos que forman el conjunto de discusiones, a modo de conclusión (Rivas, 1990). Además, se añaden los datos de la mencionada herramienta del foro virtual, como ampliación básica de la información, donde se obtuvieron 38 respuestas, en el curso 2020/2021, a la pregunta: ¿Por qué creéis que la educación patrimonial no tiene cabida en la enseñanza pre-universitaria ni en la universitaria?

Para dar validez a la investigación que ahora se presenta es necesario comparar los datos 
obtenidos a través de diferentes instrumentos, principalmente aquellos cuyas características resulten significativas (Costilla, 2013), como así hemos hecho, para obtener la necesaria triangulación, considerando igualmente como método de validez la transferibilidad, puesto que esta investigación consideramos que se puede trasladar a otros contextos, en base a los métodos de investigación empleados, y a la triangulación efectuada (Figueroa, Lizarzaburu y Valarezo, 2015).

\section{RESULTADOS}

La pregunta que inicia el cuestionario es: antes de comenzar el grado que estás cursando, ¿has recibido algún tipo de formación sobre patrimonio? En el curso 2019/2020 (en adelante, primer curso), un 54,4\% dijo que no; en el curso 2020/2021 (en lo sucesivo, segundo curso), un 54,2\% dijo que no. Como vemos, las tendencias se repiten, y gracias a la etnografía virtual, atenderemos a algunas razones para que ello sea así, a través de los resultados legislativos.

Definir el concepto de patrimonio es la pregunta abierta, única del cuestionario, que sigue a la anterior, y los resultados son llamativos. En el primer curso, hasta nueve personas no pueden dar ninguna respuesta, una persona escribe bienes con $v$, una persona, para responder, reconoce haber recurrido a Google (cuya letra inicial escribe en minúscula), una persona responde solo la palabra bachiller, otra escribe nunca haber dado patrimonio... solo once definiciones se aproximarían a la realidad, con lo cual habría más definiciones incorrectas que acertadas. En el segundo curso, solo dos personas -ya no nueve- no pueden dar ninguna respuesta, y se encuentran hasta 21 respuestas adecuadas, lo cual es una mejora sustancial, con respecto al curso anterior.

El siguiente ítem cuestiona si el alumnado ha recibido formación alguna sobre patrimonio en su grado universitario, a lo que en el primer curso un $94,1 \%$ dice que no, por un $80,8 \%$ que responde negativamente en el segundo curso. Cuando, en la siguiente pregunta, se afina, preguntando si han recibido suficiente formación, en el primer curso se afirma en el 100\% de las respuestas, que no, y en el segundo, en un $97,3 \%$ de los casos.

Definiéndose, en el cuestionario, las competencias en educación patrimonial como el adquirir la habilidad de aplicar el método científico en el estudio, conservación y gestión del Patrimonio, y preguntando si, en el grado, se ha recibido formación sobre ello, en el primer curso la respuesta recibe un $97,1 \%$ de respuestas negativas, y en el segundo curso un $91,8 \%$ en el mismo sentido. Cuando, al igual que en el párrafo anterior, se ajusta la pregunta añadiendo la calificación de suficiente, de nuevo tenemos un $100 \%$ de noes en el primer curso, y un $91,8 \%$ en el segundo.

La formación sobre patrimonio documental está ausente en el $94,1 \%$ de los casos en el primer curso, y si aplicamos el citado suficiente, en el 97,1\% de los casos; en el segundo curso, para los mismos conceptos, los porcentajes son del 90,4\% y del 97,3\%. En la misma línea y mismas preguntas, en este caso, sobre patrimonio cultural, los porcentajes, en el mismo orden previo, son del $82,4 \%$ y $92,6 \%$, y del $71,2 \%$ y $84,9 \%$, observándose mejoras de bastantes puntos de diferencia en esta cuestión concreta.

Los porcentajes vuelven a subir ostensiblemente, pues la formación sobre patrimonio inmaterial, en el primer curso, está ausente en el $91,8 \%$, y no es suficiente en el $97,1 \%$, frente a porcentajes del $91,8 \%$ (idéntico) y del $95,9 \%$ en el segundo curso. En el primer curso, alcanzamos un 
pleno doble del $100 \%$ en la ausencia de la formación sobre patrimonio industrial, frente a un $94,5 \%$, y un $98,6 \%$ de ausencia, en este caso, de formación suficiente.

Una pequeña reducción porcentual encontramos cuando se pregunta sobre patrimonio medioambiental, estando ausente, en lo que a formación se refiere, en un $89,7 \%$ durante el primer curso, y en un $97,1 \%$ no siendo suficiente. En el segundo curso, los porcentajes son menores, de un $80,8 \%$ y $91,8 \%$.

Sobre el patrimonio relacionado con las mujeres, los porcentajes, dentro de las tendencias negativas en todo momento, se reducen un tanto: en el primer curso, durante su grado, un $83,8 \%$ no había recibido formación sobre ello, y un $91,2 \%$ no había recibido formación suficiente. En el segundo curso, la diferencia es considerable: un 63\% no había recibido formación, y un $82,2 \%$ no había recibido formación suficiente, con lo cual en este apartado si hay diferencias estadísticamente significativas, de más de veinte puntos entre el $83,8 \%$ y el $63 \%$ mencionados.

El patrimonio relacionado con el colectivo LGTBI (lesbianas, gais, transgénero, bisexuales e intersexuales) es algo sobre lo que no habían recibido formación las y los estudiantes encuestados/as durante el curso $2019 / 2020$ un $88,2 \%$, siendo insuficiente en un $94,1 \%$; en el segundo curso, de nuevo, diferencias significativas: un $69,9 \%$ y un $89 \%$, de nuevo, casi 20 puntos en el primer caso, considerándose en ambos cursos la formación como insuficiente, por abrumadora mayoría.

Preguntado el alumnado sobre si conocía legislación sobre patrimonio, en el primer curso, un $94,1 \%$ no conocía, y en el segundo curso, un $87,7 \%$ no conocía. En el primer curso, preguntados y preguntadas por la existencia, en el grado que están cursando, de alguna asignatura específica sobre patrimonio, un $100 \%$ de estudiantes del primer curso afirmo que no es así, mientras que un $95,9 \%$ contestó lo mismo durante el segundo curso.

Como resultados de las últimas preguntas del cuestionario atendidas, durante el primer curso, un $94,1 \%$ no había realizado algún trabajo de investigación sobre patrimonio, y un 98,5 no tenía previsto realizar un trabajo de investigación sobre Patrimonio; durante el segundo curso, un $93,2 \%$ y un $94,5 \%$, respectivamente.

La pretensión de efectuar un análisis didáctico nos hace ir más allá, hasta la causa de los resultados, para lo cual atendemos a la Ley Orgánica 2/2006, de 3 de mayo, de Educación. En ella, la educación patrimonial no existe como tal, más allá del artículo 23, objetivos de la enseñanza secundaria obligatoria, donde, en el antepenúltimo de ellos -letra j-, se afirma que dicha enseñanza contribuirá a desarrollar en los alumnos y las alumnas las capacidades que les permitan conocer, valorar y respetar los aspectos básicos de la cultura y la historia propias y de los demás, así como el patrimonio artístico y cultural. En la recientemente derogada Ley Orgánica 8/2013, de 9 de diciembre, para la mejora de la calidad educativa, ni patrimonio ni educación patrimonial son mencionados.

En el Real Decreto 1105/2014, de 26 de diciembre, por el que se establece el currículo básico de la Educación Secundaria Obligatoria (ESO) y del Bachillerato, la expresión educación patrimonial no se ve reflejada. Sin embargo, patrimonio lo encontramos 43 veces, patrimoniales quince, y patrimonial en cinco ocasiones; pese a ello, las concreciones son mínimas en la ESO: en Lengua Castellana y Literatura, como conocimiento de la lengua como patrimonio histórico y cultural; 
en Educación Plástica, Visual y Audiovisual, sobre lenguajes visuales; en Música, sobre patrimonio musical español...

Puede que hubiera quien albergara esperanzas en la nueva legislación. Pues bien, la Ley Orgánica 3/2020, de 29 de diciembre, por la que se modifica la Ley Orgánica 2/2006, de 3 de mayo, de Educación, en sus 86 páginas, no hace ni la más mínima mención a la educación patrimonial.

Profundizamos en los resultados para comprender un resultado específico, pues cuando se preguntaba si, en el grado que estaban cursando, existía alguna asignatura específica sobre patrimonio, en el primer curso el $100 \%$ afirmaba que no, pero en el segundo curso la respuesta no era unánime, sino de un $95,9 \%$.

La etnografía virtual se torna en excelente herramienta para visualizar nuevos resultados que hagan de esta investigación un verdadero análisis didáctico. Recurrimos a las fuentes primarias, en este caso, a los planes de estudio, y a su aprobación general a través de las memorias de verificación de todos los títulos en la Facultad de Ciencias de la Educación de Málaga, obteniendo los siguientes resultados.

Comenzamos por los estudios cuyo alumnado contestó al cuestionario, y en este caso, por el Doble Grado en Educación Primaria y Estudios Ingleses. En la fecha en que se finalizaba la presente investigación, en la web de la Universidad de Málaga, no se encontraba la memoria de verificación del citado doble grado, por lo cual recurrimos, en el mismo espacio, a las memorias del Grado en Estudios Ingleses, y del Grado en Educación Primaria (Universidad de Málaga, 2021a). En la primera de ellas, del Grado en Estudios Ingleses, aunque encontremos Literatura, Historia de la Lingüística, Lengua Clásica, o Fuentes Grecolatinas en la Lengua y Cultura Inglesas, por citar algunas, no hay mención expresa a la educación patrimonial. Atendiendo a la Memoria de Verificación del Título del Grado en Educación Primaria, contemplaremos dicho grado, así como el doble grado también. En ella, lo más aproximado que encontramos, es una asignatura de cuarto curso, optativa, llamada Didáctica del Patrimonio y la Cultura Andaluza, en la cual se abordan el patrimonio cultural andaluz y las organizaciones supraestatales, la Ley Andaluza de Patrimonio, los Bienes de Interés Cultural en Andalucía, los espacios patrimoniales en Andalucía, el Patrimonio de la Humanidad (en el cual incluyen flamenco, dieta mediterránea, espacios naturales y patios), las fiestas y tradiciones populares, la proyección educativa de la cultura andaluza, el análisis del currículum de Educación Primaria y su relación con el patrimonio andaluz, la educación patrimonial y el patrimonio cultural, etcétera (Universidad de Málaga, 2020a).

Con respecto al segundo grado analizado, Educación Social, la Memoria de Verificación del Título nos mencionaba una asignatura, de nuevo optativa, cuyo nombre era Actividades y dinámicas para el ocio y el tiempo libre en expresión plástica, semestral, de seis créditos, como la anterior de Educación Primaria (Universidad de Málaga, 2021b). En ella, se atendería a la interpretación del patrimonio, museos, y centros de arte. Sin embargo, cuando nos dirigimos a la planificación docente del grado, esta asignatura ya no existe (Universidad de Málaga, 2020b).

En el penúltimo, en base a nuestro orden de análisis, de los estudios de grado de la Facultad de Ciencias de la Educación, el Grado en Pedagogía, no encontramos referencia alguna a la educación patrimonial (Universidad de Málaga, 2021c). Por último, en el Grado en Educación Infantil, 
en su Memoria de Verificación del título (Universidad de Málaga, 2021d), hallaríamos, como mayor aproximación a nuestro objeto de estudio, la asignatura optativa, de cuarto curso, y de seis créditos, que forma parte del título de Declaración Eclesiástica de Competencia Académica otorgado por la Conferencia Episcopal Española para los maestros y las maestras de Educación Infantil, Ilamada Religión, Cultura y Valores. Aunque en la citada Memoria, se mencionan en el temario de la asignatura cuestiones acordes con nuestra investigación, como la influencia del cristianismo en nuestro patrimonio cultural, o el concepto de historia en el judaísmo, cristianismo, e islam, en la guía docente de la asignatura del curso 2020/2021 dichos asuntos no se abordan explícitamente, siendo la temática fundamental la reflexión y el diálogo sobre la trascendencia humana, el origen, desarrollo y características del hecho religioso en relación con las culturas y el diálogo interreligioso e intercultural, así como la iniciación al conocimiento de la Biblia; donde se mencionaba la cuestión patrimonial, en la guía docente se encuentra el tema llamado las ciencias de la religión; donde se podía encontrar el concepto de historia en distintas religiones, encontramos el tema denominado el hecho religioso cristiano y la cultura (Universidad de Málaga, 2020c).

Conocidos los resultados de los cuestionarios, así como la realidad de lo que es posible encontrar en las memorias de verificación de todos los grados de la Facultad de Ciencias de la Educación de la Universidad de Málaga, nuestro último resultado es el análisis de las respuestas del alumnado encuestado, obtenidas a posteriori, con el objeto de comprender unas cifras que probablemente podrían considerarse alarmantes.

En primer lugar, encontramos un dato enormemente significativo, repetido por distintas alumnas: para quienes cursaron el bachiller científico-tecnológico, no existía, en el bachillerato, en los institutos de enseñanza secundaria, la oportunidad de elegir la asignatura optativa denominada Patrimonio Cultural y Artístico de Andalucía, una materia del bloque de asignaturas de libre configuración autonómica de $1^{\circ}$ de Bachillerato, que sí podía seleccionar el alumnado de bachillerato de humanidades y ciencias sociales. El hecho de no constituirse en una materia troncal creemos que ya significa mucho.

Las cuestiones metodológicas, por parte del alumnado que sí estuvo matriculado en dicha asignatura de Patrimonio Cultural, son constantes, e influyen en estos resultados, constituyéndose, de hecho, en resultado mismo. Consideran haber aprendido más bien poco (lo cual queda absolutamente corroborado con los resultados de los cuestionarios), en algún caso, hasta habiéndose encontrado redactando leyes dictadas por el profesorado, como dinámica de clase. Algún alumno llega a denominar la materia como la típica asignatura optativa en la que no se hacía prácticamente nada, por lo que el alumnado se matriculaba para tener una o dos horas libres a la semana, encontrándose la asignatura con menos de quince estudiantes en total para todo un instituto en un curso concreto. Otra alumna, de centro de enseñanza privado, habla de un temario extenso, con calificaciones en base a exámenes poco complicados. Por último, dentro de un alto número de testimonios, un alumno consideraba que todo acababa en un examen final tipo test de la materia cada trimestre y, aunque se les mostraron muchas fiestas y repasaron tradiciones, se convirtió en una asignatura que al final no se disfrutó por el hecho de que fue pura teoría, y un examen final.

Un nuevo asunto tratado en repetidas ocasiones es la inclusión del patrimonio en la 
asignatura de Historia del Arte. Una alumna consideraba que aprendieron sobre numerosas obras de arte que son consideradas patrimonio de la humanidad y, a pesar de que tuvieran que aprender innumerables fechas y un alto número de características distintas, fueron clases amenas. Otras estudiantes estimaban que apenas podían detenerse con calma en un periodo artístico concreto porque no les daba tiempo: una obra podía fascinarles, pero su análisis era efímero; los temarios se impartieron con gran rapidez, hecho agravado por la cuarentena que supuso la pandemia. Una última reflexión, apropiada a nuestro entender, es la de una alumna que consideraba que a lo largo de los años creía que habían recibido formación sobre patrimonio cultural: en Lengua y Literatura se incluían obras literarias, autores, audiovisuales, etc.; en Geografía, aprendían sobre lugares y paisajes naturales; en Historia del Arte, veían obras de arte como cuadros, esculturas y edificios. Sin embargo, se enseñaban en base a memorizar, y muchas de estas asignaturas se transformaban en monótonas y aburridas, lo que hacía que el alumnado no se viera atraído por ellas cuando, en realidad, se enseñan las temáticas eran muy interesantes sobre nuestro propio entorno. Además, destacan que se trata todo muy brevemente, no se indaga, y la enseñanza se conforma solo con los datos más superficiales.

\section{DISCUSIÓN}

La difusión del patrimonio es el mejor medio para adquirir conciencia de nuestra identidad colectiva, y constituye una condición para su conservación (Sánchez, 2001). Comprender el pasado para entender el presente, así como ubicarnos en el presente siendo conscientes de dónde venimos, es básico (Nadal, 2020). Porcentajes superiores al 90\% en un alto número de cuestiones abordadas nos aportan información inequívoca.

Las cifras suministran un criterio objetivo para poder juzgar un acontecimiento (Gordon, 2012), y es fundamental para el vigente análisis considerar las estimaciones del alumnado de la Facultad de Ciencias de la Educación de la Universidad de Málaga, cuyas valoraciones ofrecen unas cifras de una contundencia considerable. El análisis de los datos se centraba en los sujetos, pues su objetivo es comprender a las personas, en su contexto social (Mejía, 2011), y atendiendo a este. Es por ello que debíamos ir más allá, para que nuestra investigación fuera realmente didáctica.

Como considera el Ministro de Universidades del Estado español y uno de los autores más citados del mundo en ciencias sociales, Manuel Castells Oliván, los resultados obtenidos deseamos que sean útiles para aprender a pensar a partir de la práctica y para la práctica, pues solo la relación con la práctica social verifica las teorías sociales y las investigaciones científicas (Castells y De Ipola, 1981). Sin duda, los resultados prácticos indican que el alumnado no considera tener formación sobre educación patrimonial, de forma inequívoca.

Precisamente, la legislación actual referida a la enseñanza no presta atención a la educación patrimonial, lo cual puede comprobar cualquier investigador o investigadora. Por supuesto, un alto número de contenidos entrarían dentro de la conceptualización periférica de patrimonio, pero coincidimos en que, bajo parámetros académicos, nuestra definición va más allá de un encaje superfluo, y hablamos de educación desde y hacia el patrimonio, y ya contamos con investigaciones análogas en las que encontramos que, de nuevo, en la Universidad de Málaga, y en este caso en el 
Grado en Historia, la mayoría del alumnado no define correctamente el concepto de patrimonio, y no existe una asignatura con el perfil directo de ello en la citada carrera universitaria (Fernández, Fernández y Medina, 2020).

Que la legislación no acompañe, en este caso, no significa que impida, tanto en la enseñanza primaria, como secundaria y universitaria. Que no existan materias específicas, salvo la mencionada optativa, del Grado en Educación Primaria, de Didáctica del Patrimonio y la Cultura Andaluza, no significa que no puedan crearse. El alumnado ha expresado con total claridad sus carencias formativas. Quienes gestionan los contenidos de la enseñanza debieran considerarlo, mientras que el profesorado, y la comunidad educativa en sí, por su parte, debe construir sus espacios, tenerlos en propiedad colectiva, y autogestionarlos, como uno de tantos métodos para desarrollar una auténtica educación patrimonial, cuya ausencia no debiera ser algo coincidente en futuras investigaciones análogas.

\section{REFERENCIAS}

Arjones, A. (2017). La Educación y el Patrimonio Industrial de España: la protección del patrimonio industrial a través de la competencia en conciencia y expresiones culturales. En Martínez, J. C. (coord.), II Congreso Internacional Virtual sobre la Educación en el siglo XXI del 15 al 29 de marzo de 2017 (pp. 224-232). Grupo Eumed.net. https://www.eumed.net/librosgratis/actas/2017/educacion/20-la-educacion-y-el-patrimonio-industrial.pdf

Cambil, M. E. y Tudela, A. (2017). Educación y patrimonio cultural. Fundamentos, contextos y estrategias didácticas. Ediciones Pirámide.

Carrión, A. (coord.) (2015). Plan Nacional de Educación y Patrimonio. Ministerio de Educación, Cultura y Deporte.

Castells, M. y De Ipola, E. (1981). Metodología y Epistemología de las Ciencias Sociales. Ayuso.

Clarembeaux, M. (2010). Educación en cine: memoria y patrimonio. Comunicar, 18(35), 25-32. https://doi.org/10.3916/C35-2010-02-02

Comunidad de Madrid (2021). Plan de Educación Patrimonial de la Comunidad de Madrid. https://www.comunidad.madrid/cultura/patrimonio-cultural/plan- educacion-patrimonial comunidad-madrid

Costilla, D. (2013). Análisis de la problematización en la metodología cualitativa mediante la triangulación de instrumentos. Atlante, enero 2013, 1-12. https://www.eumed.net/rev/atlante/2013/01/problematizacion-metodologia.html

De la Hoz, A. (2021). Educación y Patrimonio. Metodologías y Buenas Prácticas. UNESCO Etxea.

Escolano, A. (2010). La cultura material de la escuela y la educación patrimonial. Educatio Siglo XXI, 28(2), 43-64. https://revistas.um.es/educatio/article/download/111961/106281/0

Fernández, A. R., Fernández, M. y Medina, I. R. (2020). La evaluación de las competencias en Educación Patrimonial del alumnado del Grado de Historia de la Universidad de Málaga. International Journal of New Education, 6, 2-17. https://doi.org/10.24310/IJNE3.2.2020.8451

Figueroa, A. E., Lizarzaburu, A. S. y Valarezo, A. (2015). Validez interna y externa de la investigación cualitativa. Revista Contribuciones a las Ciencias Sociales, 30 , s. p. 
http://www.eumed.net/rev/cccss/2015/04/transferibilidad.html

Feyerabend, P. (2001). Cómo defender a la sociedad de la ciencia. Polis, 1, 1-8. https://dialnet.unirioja.es/descarga/articulo/2797156.pdf

Fontal, O. e Ibañez-Etxeberria, A. (2017). La investigación en Educación Patrimonial. Evolución y estado actual a través del análisis de indicadores de alto impacto. Revista de Educación, 375, 184-214. https://doi.org/ 10.4438/1988-592X-RE-2016-375-340

Fontal, O., García, S., Arias, B. y Arias, V. B. (2019). Assessing the Quality of Heritage Education Programs: Construction and Calibration of the Q-Edutage Scale. Revista de Psicodidáctica, 24(1), 31-38. https://doi.org/10.1016/j.psicod.2018.07.003

Foucault, M. (2019). Microfísica del poder. Siglo XXI.

Freire, P. (2011). La educación como práctica de la libertad. Siglo XXI.

Gordon, V. (2012). Los orígenes de la civilización. Fondo de Cultura Económica.

Hine, C. (2004). Etnografía Virtual. Editorial UOC.

Ibañez, A., Fontal, O. y Cuenca, J. M. (2015). Actualidad y tendencias en Educación Patrimonial. Educatio Siglo XXI, 33(1), 11-14. https://revistas.um.es/educatio/article/view/222471

Instituto Andaluz del Patrimonio Histórico. (2021). Primer MOOC sobre Patrimonio Cultural de Andalucía.https://www.juntadeandalucia.es/organismos/culturaypatrimoniohistorico/iaph/actu alidad/noticias/detalle/254621.html

Lacomba, J. A. (2011). Sobre la historia y su función en la sociedad. En VVAA, La recuperación de la memoria histórico-educativa andaluza. Editorial Sarriá.

Ley 16/1985, de 25 de junio, del Patrimonio Histórico Español. https://www.boe.es/buscar/act.php?id=BOE-A-1985-12534

Ley Orgánica 2/2006, de 3 de mayo, de Educación. https://www.boe.es/eli/es/lo/2006/05/03/2

Ley Orgánica 3/2020, de 29 de diciembre, por la que se modifica la Ley Orgánica 2/2006, de 3 de mayo, de Educación. https://www.boe.es/eli/es/lo/2020/12/29/3

Ley Orgánica 8/2013, de 9 de diciembre, para la mejora de la calidad educativa. https://www.boe.es/eli/es/lo/2013/12/09/8/con

Mejía, J. (2011). Problemas centrales del análisis de datos cualitativos. Revista Latinoamericana de Metodología de la Investigación Social, $\quad 1, \quad 47-60$. https://dialnet.unirioja.es/descarga/articulo/5275948.pdf

Ministerio de Cultura y Deporte. (2021). Actuaciones. http://www.culturaydeporte.gob.es/planesnacionales/planes-nacionales/educacion-y-patrimonio/actuaciones.html 27-4-2021

Nadal, A. (2020). Análisis didáctico-contextual: España, 1780-1814. Revista Contribuciones a las Ciencias Sociales, septiembre 2020, 1-11. https://www.eumed.net/rev/cccss/2020/09/analisisdidactico-contextual.html

Palomero-llardia, I. (2020). Investigación-acción en la enseñanza de la historia: simulación arqueológica en entornos educativos formales. Psychology, Society, \& Education, 12(2), 259273. https://doi.org/ 10.25115/psye.v10i1.2467

Real Decreto 1105/2014, de 26 de diciembre, por el que se establece el currículo básico de la Educación

Secundaria

Obligatoria

$y \quad$ del

Bachillerato. 
https://www.boe.es/buscar/act.php?id=BOE-A-2015-37\&tn=6

Rivas, J. I. (1990). Investigación naturalista en educación. Promolibro.

Sánchez, C. (coord.). (2001). El Patrimonio Industrial en Andalucía. Junta de Andalucía. Consejería de Cultura.

Taylor, S. y Bogdan, R. (2002). Introducción a los métodos cualitativos de investigación. Editorial Paidós.

Universidad de Málaga. (2020a). Didáctica del Patrimonio y la Cultura Andaluza. Recuperado el 25 de abril de 2021 de https://www.uma.es/centers/subject/facultad-de-ciencias-de-laeducacion/5010/51462/

Universidad de Málaga. (2020b). Lista de Asignaturas de Graduado/a en Educación Social. Recuperado el 25 de abril de 2021 de https://www.uma.es/centers/subjects _center/facultad-de-ciencias-de-la-educacion/5060/

Universidad de Málaga. (2020c). Religión, Cultura y Valores. Recuperado el 25 de abril de 2021 de https://www.uma.es/centers/subject/facultad-de-ciencias-de-la-educacion/5009/53097/

Universidad de Málaga. (2021a). Doble Grado en Educación Primaria y Estudios Ingleses. Recuperado el 25 de abril de 2021 de https:/www.uma.es/ep-eeii/info/118771/ep-eeii-inicio/

Universidad de Málaga. (2021b). Grado en Educación Social. Recuperado el 25 de abril de 2021 de https://www.uma.es/grado-en-educacion-social

Universidad de Málaga. (2021c). Grado en Pedagogía. Recuperado el 25 de abril de 2021 de https://www.uma.es/grado-en-pedagogia

Universidad de Málaga. (2021d). Grado en Educación Infantil. Recuperado el 25 de abril de 2021 de https://www.uma.es/grado-en-educacion-infantil 